\title{
A self-calibrating electrometer for atmospheric charge measurements from a balloon platform
}

Article

Accepted Version

Nicoll, K. (2013) A self-calibrating electrometer for atmospheric charge measurements from a balloon platform. Review of Scientific Instruments, 84 (9). 096107. ISSN 10897623 doi: https://doi.org/10.1063/1.4821500 Available at https://centaur.reading.ac.uk/34255/

It is advisable to refer to the publisher's version if you intend to cite from the work. See Guidance on citing.

Published version at: http://dx.doi.org/10.1063/1.4821500

To link to this article DOI: http://dx.doi.org/10.1063/1.4821500

Publisher: AIP

All outputs in CentAUR are protected by Intellectual Property Rights law, including copyright law. Copyright and IPR is retained by the creators or other copyright holders. Terms and conditions for use of this material are defined in the End User Agreement.

$\underline{\text { www.reading.ac.uk/centaur }}$ 
Central Archive at the University of Reading

Reading's research outputs online 


\title{
Note: A self-calibrating electrometer for atmospheric charge measurements from a balloon platform
}

\author{
K. A. Nicoll \\ Department of Meteorology, University of Reading, Reading, Berkshire, RG6 6BB, UK \\ (Received XXXXX; accepted XXXXX; published online XXXXX) \\ (Dates appearing here are provided by the Editorial Office)
}

Charged aerosol particles and water droplets are abundant throughout the lower atmosphere, and may influence interactions between small cloud droplets. This note describes a small, disposable sensor for the measurement of charge in nonthunderstorm cloud, which is an improvement of an earlier sensor ${ }^{1}$. The sensor utilizes a self-calibrating current measurement method. It is designed for use on a free balloon platform alongside a standard meteorological radiosonde, measuring currents from $2 \mathrm{fA}$ to $15 \mathrm{pA}$ and is stable to within $5 \mathrm{fA}$ over a temperature range of $5^{\circ} \mathrm{C}$ to $-60^{\circ} \mathrm{C}$. During a balloon flight with the charge sensor through a stratocumulus cloud, charge layers up to $40 \mathrm{pCm}^{-3}$ were detected on the cloud edges.

Away from the strong electrification of thunderstorms, charge is concentrated in the atmosphere in layers of aerosol such as Saharan dust ${ }^{2,3}$ and volcanic ash ${ }^{4,5}$, and on cloud droplets ${ }^{6}$. Charge is particularly prevalent on the edges of layer clouds which are horizontally extensive, as a consequence of current flow in the global atmospheric electric circuit. The vertical gradient in conductivity at a cloud edge generates space charge at the upper and lower cloud boundaries, which may influence cloud microphysical processes such as the collection of charged particles by droplets, and droplet-droplet collisions ${ }^{7,8}$.

Measurements of charge in non-thunderstorm cloud have previously been made from a free balloon platform by measuring the rate of change of voltage on a small sensing electrode ${ }^{1}$, where the voltage change is due to current flow between the atmosphere and the electrode due to the vertical motion of the sensor. The voltage measurement method was initially implemented over the direct measurement of current to avoid previously reported problems with poor thermal stability in the large feedback resistors used for the current measurement ${ }^{9,10}$. Although the voltage measurement technique has a low thermal drift, it is prone to saturation, leading to lock up. This note describes an improved charge sensor, which measures current directly, and is compensated against drift by the implementation of a T-network in the feedback resistor stage, and applying a regular standardized injection of a known calibration current pulse. The electrometer is disposable and designed for use alongside a standard meteorological radiosonde flown from a free balloon platform with the Vaisala RS92 radiosonde, using the PANDORA interface ${ }^{11}$. This allows the electrometer data to be transmitted via the radio link at $1 \mathrm{~Hz}$, synchronously with the meteorological data. The use of a free balloon radiosonde platform allows high vertical resolution cloud charge measurements $(\sim 5 \mathrm{~m})$ at low cost.

The electrometer comprises a single sensing electrode, connected to an electrometer circuit measuring the electrode current. The sensing electrode responds to changes in charge, either induced i.e. due to changes in electric field, or from impaction of charged particles. The sensing electrode is a brass sphere of diameter $12 \mathrm{~mm}$, connected by a $1 \mathrm{M} \Omega$ protection resistor (through a Polytetrafluoroethylene (PTFE) sheath) to the electrometer circuit, as previously ${ }^{1}$. The electrometer is flown in a small electrically shielded box (to minimize interference from the radiosonde system) with dimensions $6.5 \times 12 \times 4 \mathrm{~cm}$.

The electrometer circuit is shown in Figure 1, and consists of a current to voltage converter, IC1a, with a Tnetwork of resistors. To achieve the sensitivity required to measure charge in the fair weather atmosphere with a typical current to voltage converter circuit, an effective resistance of $10^{12} \Omega$ is required in the feedback stage. The use of such high value resistors, which can drift significantly with temperature ${ }^{10}$, is undesirable for a balloon borne sensor which will experience a wide range of temperature changes, typically from $+20^{\circ} \mathrm{C}$ to $-60^{\circ} \mathrm{C}$. A $\mathrm{T}$-network minimizes the need for high value resistors by synthesizing the total resistance with lower value resistors. The equivalent feedback resistance of the T-network (R1, $\mathrm{R} 2, \mathrm{R} 3$ ) is $0.5 \mathrm{~T} \Omega$. A $4 \mathrm{~V}$ ref (LM4040 4.1V) ensures a positive output voltage, with bipolar current inputs, as required by the PANDORA interface. A 4053 changeover switches between measurement and current calibration modes. In measurement mode, IC5a is configured in voltage follower mode, ensuring that the electrometer circuit responds only to currents flowing to the electrode. In current calibration mode, a known current is generated by applying a reference voltage (from IC4 and through IC2) to an integrator stage, to the electrometer circuit input (IC1a). Positive and negative currents are generated (the two IC2 stages provide symmetrical equal and opposite voltages, $V_{\text {ref }}= \pm 25 \mathrm{mV}$ ), using this ramp generator technique ${ }^{12}$, fixed at $\pm 2.5 \mathrm{pA}$. The calibration current, $i_{\text {cal }}$ is given by 


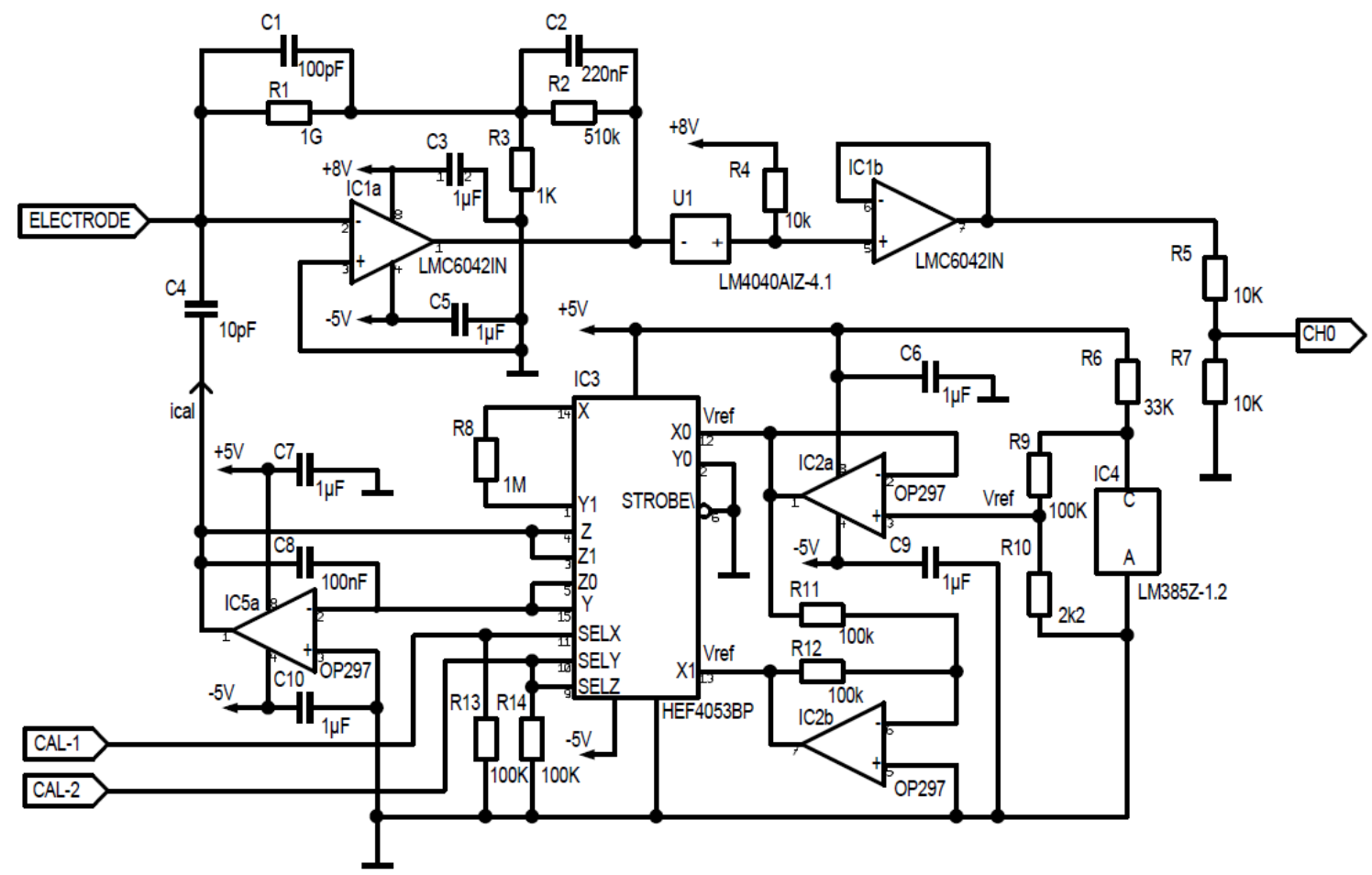

Figure 1. Schematic for the self-calibrating electrometer showing the electrometer (current to voltage converter) IC1a and output offset buffer; voltage reference generator IC4/IC2; integrator ramp voltage generator IC5; and change over switches IC3. IC3 consists of 3 separate switches, each of which has two independent inputs (X0, X1; Y0, Y1; Z0, Z1), a common input (X,Y, Z), and select inputs(SELX, SELY, and SELZ).

$$
i_{c a l}=\frac{C_{4}}{C_{8}} \frac{V_{r e f}}{R_{8}}
$$

The transition from measurement to current calibration mode is made via an HEF4053 changeover switch (IC3), which contains three separate changeover switches. The three switches toggle between measurement mode, positive current calibration mode and negative current calibration mode. These are selected by two separate $+5 \mathrm{~V}$ pulses from a 16F676 microcontroller on the PANDORA from CAL-2 to initiate a positive calibration period (i.e. SELY and SELZ are taken high), and CAL-1 for a negative one (SELX, SELY and SELZ are all taken high). A measurement period lasts for 5 minutes, followed by 10 seconds of $+2.5 \mathrm{pA}$ and 10 seconds of $-2.5 \mathrm{pA}$. The relationship between the current flowing to the electrode, $i$, and the recorded output voltage, $V$, is derived from the known calibration currents. For each 5 minute measurement period, a linear fit is made between the mean output voltage during the positive and negative calibration periods (mean of 10 points), against the known calibration currents of $+2.5 \mathrm{pA}$ and $-2.5 \mathrm{pA}$ for the positive and negative calibration periods respectively. This yields an expression of the form $i=a V+b$, where typically $a \approx 2 \times 10^{-}$ ${ }^{12}$ and $b \approx-1 \times 10^{-1}$, which is applied to the output voltage, $V$, to derive the measured input current, $i$. During a typical radiosonde flight the electrometer will experience a range of atmospheric temperatures from $+20^{\circ} \mathrm{C}$ to $-60^{\circ} \mathrm{C}$, therefore it is important to assess the temperature stability of the electronic circuit. Thermal drift in the electronic circuit is expected due to changes in the T-network resistors (mainly R1) and drift in the offset voltage of the LMC6042 op amp, IC1a. The choice of the T-network resistor values means that the drift due to the poor thermal stability of the resistors has been approximately cancelled by the drift in the offset voltage. To demonstrate this, Figure 2 shows the electrometer current (in black) during a flight from $4-20 \mathrm{~km}$ in clear air conditions, when the temperature varied by $55^{\circ} \mathrm{C}$ (grey line). The stability of the electrometer in measurement mode is clear, as is the reliability of the current calibration approximately every $700 \mathrm{~m}$.

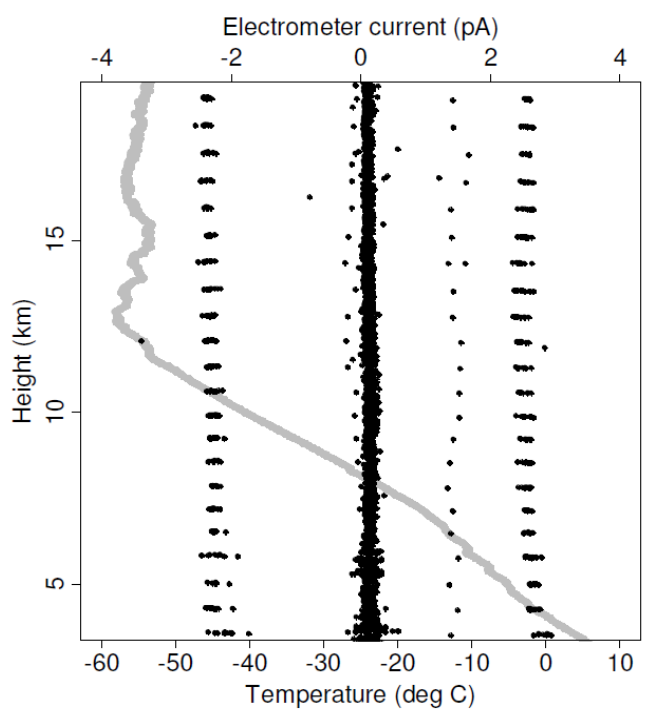

Figure 2. Vertical profile from a radiosonde flight of the electrometer from Reading, UK. The current measured by the electrometer is shown by black points and temperature measured by the RS92 radiosonde denoted by the grey line. 
(a)

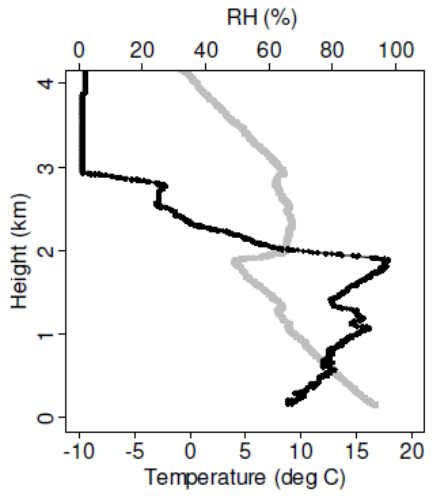

(b)

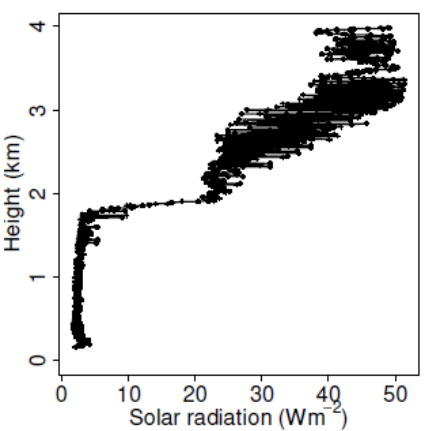

(c)

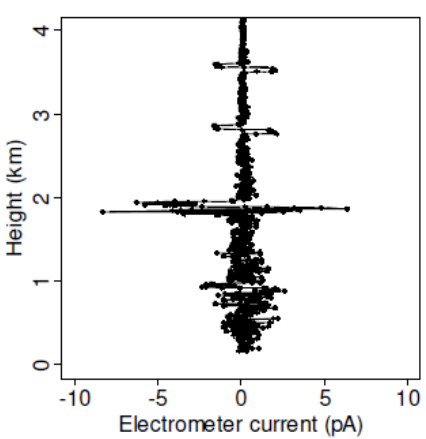

(d)

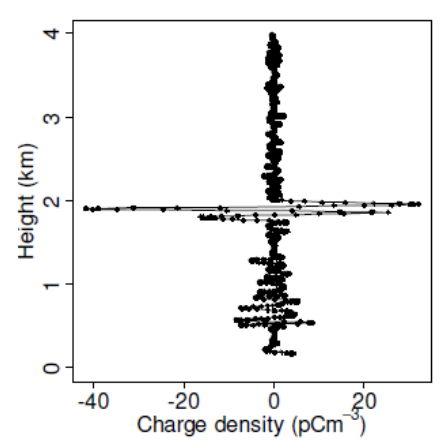

Figure 3. Vertical profiles from a radiosonde flight of the electrometer and a solar radiation sensor, through an extensive stratoculumus cloud layer on 03/07/13 over Reading, UK. (a) Temperature (grey) and relative humidity (RH, black) measured by the RS92 radiosonde, (b) measurements from a downward pointing solar radiation sensor on the same flight, (c) electrometer current, (d) charge density derived from the electrometer measurements.

From a spline fit to the electrometer current data, the drift in the output over the temperature range from 5 to $-60^{\circ} \mathrm{C}$ was $5 \mathrm{fA}$, which is negligible when compared to the typical currents generated by charge layers (of order several pA).

Several radiosonde flights of the electrometer have been made through non-thunderstorm cloud conditions from Reading, UK. Figure 3 shows a typical example of data through a layer of stratocumulus cloud. The location of the cloud layer between 1.8 and $2.1 \mathrm{~km}$ is obvious from the radiosonde's measurement of RH (Figure 3(a)), and the increase in solar radiation shown in Figure 3(b), as measured by a downward pointing solar radiation sensor $^{13}$. Figure 3(c) shows the current flowing to the electrometer, where the increased magnitude and variability of the current demonstrate the presence of charge within the cloud layer. The electrometer shows a strong response to the cloud charge, without any saturation issues. The charge density measured by the electrometer can be derived from either the recorded output voltage of the electrometer or the current calculated from the recorded output voltage. Here, the recorded output voltage is used to calculate the space charge density, using the method detailed in ${ }^{1}$. This method assumes that the majority of the changes in the output voltage of the sensor are due to induced effects (calculations show that the typical response to impacted droplets is small). From ${ }^{1}$ the space charge density, $\rho$, depends on the vertical ascent rate, $w$ (found from the GPS data measured by the RS92), the rate of change of the electrometer output voltage with time, $d V / d t$, the permittivity of free space, $\varepsilon_{0}$, and the effective diameter of the sensing electrode, $d_{\text {eff }}$, taken here as $20.3 \mathrm{~mm}^{\text {from }}{ }^{1}$

$$
\rho=\frac{\varepsilon_{0}}{d_{e f f}} \frac{1}{w} \frac{d V}{d t}
$$

The charge density derived from equation (2) is shown in Figure $3(\mathrm{~d})$, demonstrating that charge of up to $\pm 40 \mathrm{pCm}^{-3}$ is present within the cloud layer, with positive charge charge at the upper cloud edge and negative charge at the base.

In summary, using the method of electrode current measurement to detect charge provides significant advantages over a previous voltage measurement approach. The circuit is stable to within 5fA over a wide range of atmospheric temperatures and does not lock up when exposed to highly charged regions. As evidenced by the flight results shown, the charge sensor is effective at detecting charge in non-thunderstorm cloud and is also expected to be suitable for charge detection in aerosol layers.

The author acknowledges the support of the Leverhulme Trust through an Early Career Fellowship and Prof. R. G. Harrison for many useful discussions. The technical staff in the Department of Meteorology prepared the electronic circuit boards and assisted with balloon launches.

${ }^{1}$ Nicoll K.A. and R.G. Harrison, Rev. Sci. Instrum., 80, 0145014 (2009).

${ }^{2}$ Nicoll K. A., R.G. Harrison, Z. Ulanowski, Environ. Res. Lett. 6, 014001 (2011).

${ }^{3}$ Gringel W, Mühleisen R, Beitraege zur Physik der atmosphaere, 51, 121-128 (1978).

${ }^{4}$ Harrison R.G., K.A. Nicoll, Z. Ulanowski, T.A. Mather, Environ. Res. Lett., 5, 024004 (2010).

${ }^{4} 5$ Kondo Y., R. Reiter , H. Jager et al, Pure Appl. Geophys. 120, 11-17 (1982).

${ }^{6}$ Beard, K. V., H. T. Ochs III, C.H. Twohy, Geophys. Res. Lett., 31, L14111, (2004).

${ }^{7}$ Tinsley, B.A, Space Sci. Revs., 94, 231-258, (2000).

${ }^{8}$ Rycroft, M.J., K.A. Nicoll, K.L. Aplin, R.G. Harrison, J. Atmos. Sol. Terr. Phys., 90-91, 198-211 (2012).

${ }^{9}$ Harrison, R.G., K.L. Aplin, Atmos. Res., 85, 199-208 (2007).

${ }^{10}$ Markson, R., L.H. Ruhnke, E.R. Williams, Atmos. Res. 51, 315-321, (1999).

${ }^{11}$ Harrison R.G., K.A. Nicoll and A.G. Lomas, Rev. Sci. Instrum., 83, 036106, (2012).

${ }^{12}$ Harrison, R.G. and K.L. Aplin, Rev. Sci. Instrum., 71, 3231 3232 (2000).

${ }^{13}$ Nicoll, K.A and R.G. Harrison, Rev. Sci, Instrum, 83, 025111, (2012). 